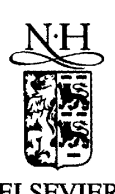

Applied Mathematics and Computation 144 (2003) 1-2

www.elsevier.com/locate/amc

\title{
A variational approach to the Burridge-Knopoff equation
}

\section{Ji-Huan $\mathrm{He}$}

LNM, Institute of Mechanics, Chinese Academy of Sciences, Beijing

College of Science, Shanghai Donghua University, P.O. Box 471, 1882 Yan'an Xilu Road,

Shanghai 200051, China

Shanghai Institute of Applied Mathematics and Mechanics, 149 Yanchang Road, Shanghai 200072,

People's Republic of China

\begin{abstract}
A variational principle is obtained for the Burridge-Knopoff model for earthquake faults, and this paper considers an analytic approach that does not require linearization or perturbation.

(c) 2002 Elsevier Science Inc. All rights reserved.

Keywords: Burridge-Knopoff model; Earthquake; Variational principle
\end{abstract}

Investigations of the dynamics of slowly driven threshold systems have triggered renewed interest in the nonlinear dynamics of earthquake faults. The governing equation describing dynamics of earthquake faults is the well-known Burridge-Knopoff equation [1-3], which reads

$$
\frac{\partial^{2} u}{\partial t^{2}}=\xi^{2} \frac{\partial^{2} u}{\partial x^{2}}-u-F\left(u_{t}\right)+v t
$$

where $u_{t}=\partial u / \partial t, u$ is the normalized displacement, $\xi$ is the normalized sound velocity, $v$ is the dimensionless driving velocity, $F$ is the dimensionless friction force.

E-mail addresses: jhhe@dhu.edu.cn, ijnsns@yahoo.com.cn (J.-H. He).

0096-3003/02/\$ - see front matter @ 2002 Elsevier Science Inc. All rights reserved. doi:10.1016/S0096-3003(02)00379-X 
Adomian [3] applied the decomposition method to the solution of the Burridge-Knopoff equation, in this paper, by the semi-inverse method [4,5], we obtain the following variational principle:

$$
J(u)=\iint\left\{\frac{1}{2}\left(\frac{\partial u}{\partial t}\right)^{2}-\frac{1}{2} \xi^{2}\left(\frac{\partial u}{\partial x}\right)^{2}-\frac{1}{2} u^{2}+\Phi\left(u_{t}\right)+v u t\right\} \mathrm{d} x \mathrm{~d} t
$$

where $\Phi$ is calculated by the relation

$$
\frac{\partial}{\partial t}\left(\frac{\partial \Phi}{\partial u_{t}}\right)=F\left(u_{t}\right)
$$

In view of finite element method [6], the displacement $u$ is approximated as

$$
u(t, x)=\sum_{i=1}^{N} N_{i} u_{i}
$$

and

$$
\frac{\partial u}{\partial t}=\sum_{i=1}^{N}\left(\frac{\partial u_{i}}{\partial t} N_{i}\right)
$$

The variational equation (2) provides a more complete theoretical basis not only for the finite element applications [6], but also for many direct variational methods such as Ritz's, Trefftz's and Kantorovitch's methods. We can easily obtain an oscillatory mode by the Ritz's method.

\section{References}

[1] P.G. Akishin, M.V. Altaisky, I. Antoniou, A.D. Budnik, V.V. Ivanov, Burridge-Knopoff model and self-similarity, Chaos, Solitions and Fractals 11 (2000) 207-222.

[2] P. Hahner, Y. Drossinos, Dynamics of a creep-slip model of earthquake faults, Physica A 260 (1998) 391-417.

[3] G. Adomian, The Burridge-Knopoff model, Applied Mathematical and Computation 77 (1996) $131-132$.

[4] J.H. He, Semi-inverse method of establishing generalized variational principles for fluid mechanics with emphasis on the turbomachinery aerodynamics, International Journal of Turbo \& Jet-Engines 14 (1) (1997) 23-28.

[5] J.H. He, Variational theory for linear magneto-electro-elasticity, International Journal of NonLinear Science and Numerical Simulation 2 (4) (2001) 309-316.

[6] G.L. Liu, A new finite element with self-adapting built-in discontinuity for shock-capturing in transonicflow, International Journal of Non-Linear Science and Numerical Simulation 1 (1) (2000) 25-30. 